\title{
AN ASYMPTOTIC SOLUTION FOR SHORT-TIME TRANSIENT HEAT CONDUCTION BETWEEN TWO DISSIMILAR BODIES IN CONTACT
}

\author{
By H. G. GEORGIADIS $\uparrow$ \\ (Mechanics Division, Naval Academy of Greece, \\ 33-35 G. Papandreou Street, 16231 Athens, Greece)
}

\section{J. R. BARBER and F. BEN AMMAR}

(Department of Mechanical Engineering and Applied Mechanics, University of Michigan, Ann Arbor, Michigan 48109-2125, USA)

[Received 10 April 1989. Revises 4 September 1989, 20 February 1990]

\section{SUMMARY}

A solution is obtained for the heat-conduction problem of two half-spaces of dissimilar materials, initially at different temperatures, brought into contact over half of their common boundary, the remainder of the interface being insulated. The solution is obtained by taking Laplace transforms in time and one space dimension and utilizing the Wiener-Hopf technique.

The results describe the local asymptotic fields near the boundary of a more general shape of contact area between the two bodies, at small values of time. It is therefore possible to develop a two-term short-time asymptotic expression for the heat exchanged between the bodies, one term being proportional to the area of the contact region and the other to its perimeter. This expression is derived in closed form except for an integral which depends on two dimensionless parameters-the ratios of the material conductivities and diffusivities.

Numerical values of the integral are presented for a wide range of these parameters.

\section{Introduction}

IN THIS paper, we shall be concerned with the problem of two conducting bodies, initially at different uniform temperatures, which are brought into contact over part of their surfaces at time $t=0$. The simplest case is the one-dimensional problem in which two conducting half-spaces, $y>0, y<0$, initially at uniform temperatures $0, T_{0}$ respectively, make perfect thermal contact over their common interface, $y=0$, for $t>0$. The solution is given by Carslaw and Jaeger (1, section 2.15 (i)). The temperature field depends on $y, t$ only and the interface, $y=0$, adopts the constant temperature

$$
T_{I}=\frac{K_{2} \kappa_{2}^{-\frac{1}{2}} T_{0}}{K_{1} \kappa_{1}^{-\frac{1}{2}}+K_{2} \kappa_{2}^{-\frac{t}{t}}},
$$

† Present address: Mechanics Division, School of Technology, Box 422, The Aristotle Unıversity of Thessalonik1, 54006, Greece. 
where $K_{j}, \kappa_{j}(j=1,2)$ denote the thermal conductivity and thermal diffusivity respectively of body $j$ and the suffixes 1,2 relate to the properties of the upper body $(y>0)$ and the lower body $(y<0)$ respectively.

The one-dimensional problem has no inherent length scale and hence the solution can be cast in self-similar form, in which the space and time dependence of the temperature field can be reduced to dependence on the single dimensionless parameter

$$
Y=\frac{y}{\sqrt{ }\left(\kappa_{j} l\right)} .
$$

It also follows that there is no characteristic time scale for the process and hence that there is no short- or large-time solution, nor does the process reach a steady state. The temperature field evolves continuously in time and a transition region near the interface grows in size with $t^{\frac{1}{2}}$, whilst preserving the same shape.

We also record the heat flux between the bodies, which is

$$
q_{y}=\frac{T_{0}}{\sqrt{ }(\pi t)\left\{\kappa_{1}^{\frac{1}{2}} / K_{1}+\kappa_{\frac{1}{2}}^{\frac{t}{2}} / K_{2}\right\}} .
$$

We note that the dependence of $q_{y}$ on $t^{-\frac{1}{2}}$ follows immediately from the dependence of the temperature on the single parameter $Y$.

\section{Bounded contact area}

The situation is very different if the two half-spaces are brought into contact over a bounded region, $A$, of the interface $y=0$. The contact region will have some characteristic dimension $b$, and the form of the solution will now depend on time, through the dimensionless parameters

$$
B_{j}=\frac{b}{\sqrt{ }\left(\kappa_{j} t\right)} .
$$

In particular, when both $B_{j} \ll 1$, the temperature field will approach a steady-state solution, whereas for $B_{j} \gg 1$, we anticipate a qualitatively different 'short-time' solution. In an earlier paper (2), it was shown that the short-time temperature field only differs significantly from the one-dimensional solution in regions whose minimum distance, $s$, from the boundary of the contact region satisfies the inequality

$$
S=\frac{s}{\sqrt{ }(4 \kappa, t)}<3 .
$$

Thus, for points well removed from the boundaries of $A$, the interface temperature and heat flux are given by equations (1), (3) respectively.

In the corresponding steady-state problem, the heat flux into body 1 is equal and opposite to that out of body 2 throughout the interface $y=0$ and is non-zero only in the bounded region $A$. We can therefore express the 
temperature field in body 1 in terms of this heat flux, $q(x, z)$, in the form

$$
T(x, y, z)=\frac{1}{2 \pi K_{1}} \iint_{A} \frac{q\left(x^{\prime}, z^{\prime}\right) d x^{\prime} d z^{\prime}}{\left(\left(x-x^{\prime}\right)^{2}+y^{2}+\left(z-z^{\prime}\right)^{2}\right)^{\frac{1}{2}}}, \quad y>0,
$$

where we have used the result $T=q / 2 \pi K R$ for the temperature $T$ in a half-space at a distance $R$ from a steady-state point source of strength $q$ located at the surface (1, section $2: 4)$.

By a similar argument, we find that the temperature in body 2 is

$$
T(x, y, z)=T_{0}-\frac{1}{2 \pi K_{2}} \iint_{A} \frac{q\left(x^{\prime}, z^{\prime}\right) d x^{\prime} d z^{\prime}}{\left(\left(x-x^{\prime}\right)^{2}+y^{2}+\left(z-z^{\prime}\right)^{2}\right)^{\frac{1}{2}}}, \quad y<0,
$$

where we note that $q(x, z)$ is defined to flow out of body 2 and we also have to superpose the uniform temperature $T_{0}$ to establish the correct temperature at infinity in body 2 .

It is clear from equations (6), (7) that the steady-state temperature field is geometrically similar in the two bodies in the sense that

$$
\frac{K_{1}}{K_{2}} T(x, y, z)+T(x,-y, z)=T_{0}, \quad y>0,
$$

and in particular, on setting $y=0$, we find that the interface temperature, which must be continuous between the two bodies in the region $A$, is given by

$$
T_{I}=\frac{K_{2} T_{0}}{K_{1}+K_{2}}
$$

The corresponding steady-state heat flux at the interface depends on the extent of the particular contact region $A$, but it can be shown by asymptotic arguments that $q_{y}$ will have a square-root singularity at the boundary of $A$.

Equations (1), (9) show that the contact surface $A$ is an isotherm in both the short-time and large-time limits, but the interface temperatures in the two limits differ unless the diffusivities of the two materials are identical. We therefore anticipate that the interface temperature will initially be uniform and given by (1), but that a 'boundary layer' will grow from the boundary of the region $A$ towards the interior, tending to change the interface temperature to the value (9). This paper is principally concerned with investigating the nature of this boundary layer by an asymptotic analysis of the transient temperature field in the immediate vicinity of the boundary. The heat flux in this region is of particular interest, since it will enable us to develop a two-term asymptotic solution for the total heat flux between the contacting bodies at small values of time (2).

\section{The asymptotic problem}

At very small times, when the thickness of the boundary layer is small in comparison with the linear dimensions of the contact region and the radius of 


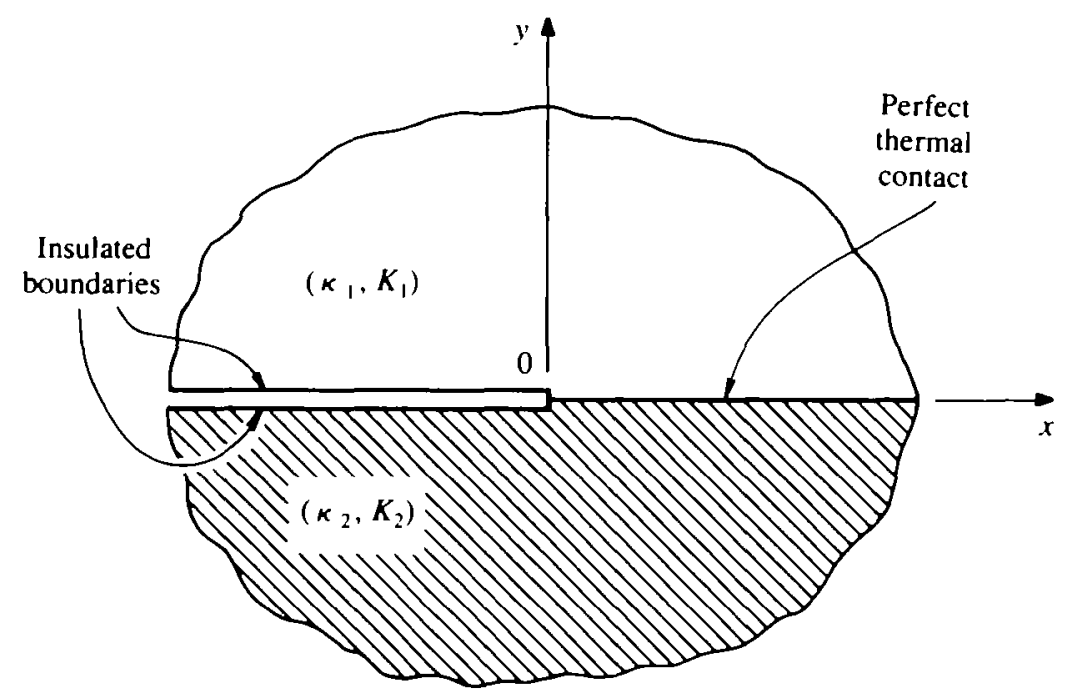

FIG. 1. Geometry of the problem. Two dissimilar half-spaces in thermal contact in the region $x>0, y=0$. The interface $x<0, y=0$ is insulated

curvature of its boundary, the local temperature field will be essentially twodimensional and can be investigated using the asymptotic method pioneered by Williams (3) in related problems of linear elasticity.

We take the $z$-axis in Cartesian coordinates to coincide with the local portion of the boundary of the contact region, in which case the boundary-layer field can be determined from the two-dimensional problem shown in Fig. 1, where the contact area $A$ consists of the half-line $x>0, y=0$.

We note that the bodies and the contact region are semi-infinite and hence that the problem has no inherent length scale. It follows that the temperature field must depend only on the parameters $X=\left(x / \sqrt{ }\left(\kappa_{j} t\right)\right), Y=\left(y / \sqrt{ }\left(\kappa_{j} t\right)\right)$ as in the one-dimensional problem introduced in section 1 above. We shall make use of this simplification at a later stage in the analysis. A large value of $X$ or $Y$ can be obtained either by making $x, y$ large or by making $t$ small. It therefore follows that the temperature field at large $X, Y$ corresponds to the short-time solution of the more general transient contact problem, whereas that at small $X, Y$ corresponds to the steady-state solution. We can therefore deduce immediately that the temperature and heat flux at the interface will tend to the values of equations (1), (3) respectively when $X$ and hence $x$ is large and positive. Also, as $X$ and $x$ tend to zero, the heat flux will exhibit a square-root singularity at the interface and the interface temperature will adopt the value of equation (9). These results are useful in determining the appropriate asymptotic behaviour of the functions in the following transform solution and hence in determining appropriate regions of convergence and analyticity. 
The governing equations in both media are written as (1)

$$
\kappa_{j} \nabla^{2} T_{j}=\frac{\partial T_{j}}{\partial t} \quad(j=1,2),
$$

where $T_{j}(x, y, t)$ are the temperatures in the two bodies and $\nabla^{2}$ is the two-dimensional Laplace operator.

The heat flux is related to the temperature through

$$
q_{y j}(x, y, t)=-K_{j} \frac{\partial T_{j}}{\partial y} .
$$

It is convenient for our integral transform approach to have zero-temperature initial conditions and this can be achieved by using the symbol $T_{2}$ to represent the difference between the temperature of body 2 and its initial uniform value $T_{0}$. With this definition, the initial and boundary conditions of the problem take the form

$$
\begin{aligned}
q_{y j}(x, 0, t) & =0, & & x<0, t>0, \\
q_{y 1}(x, 0, t) & =q_{y 2}(x, 0, t), & & x>0, t>0, \\
T_{1}(x, 0, t) & =T_{2}(x, 0, t)+T_{0}, & & x>0, t>0, \\
T_{1}(x, y, 0) & =T_{2}(x, y, 0)=0 . & &
\end{aligned}
$$

Condition (14) imposes continuity of temperature in the contact region $x>0$, $y=0$, whereas (12) states that there is no heat exchange at the interface outside the contact region.

\section{Integral transform analysis}

We introduce a one-sided Laplace transform on time and a two-sided Laplace transform to suppress the dependence on $x$. The latter transform is equivalent to the more common Fourier transform, but results in less cumbersome algebraic expressions. We write (4)

$$
\left.\begin{array}{rl}
f(x, y, s) & =\int_{0}^{\infty} f(x, y, t) e^{-s t} d t, \\
f(x, y, t) & =\frac{1}{2 \pi i} \int_{\mathbf{B r}} J(x, y, s) e^{x} d s,
\end{array}\right\}
$$

where $\mathrm{Br}$ is the Bromwich path within the strip of convergence and $\left(s^{\frac{1}{2}} p\right)$ is the complex transform parameter. 
We now apply $(16)_{1},(17)_{1}$ successively to the governing equations (10) and the initial conditions (15), obtaining the ordinary differential equations

$$
\frac{d^{2} T_{j}^{*}}{d y^{2}}(p, y, s)-s\left(\frac{1}{\kappa_{j}}-p^{2}\right) T_{j}^{*}(p, y, s)=0,
$$

which have the following solutions bounded at $y \rightarrow \pm \infty$ for medium 1,2 respectively:

$$
\left.\begin{array}{ll}
T_{1}^{*}(p, y, s)=A(p, s) e^{-\gamma_{1} y} & (y>0), \\
T_{2}^{*}(p, y, s)=B(p, s) e^{\gamma_{2} y} & (y<0),
\end{array}\right\}
$$

where

$$
\gamma_{j}=s^{\frac{1}{2}}\left(b_{j}^{2}-p^{2}\right)^{\frac{1}{2}}, \quad b_{j}=\kappa_{j}^{-\frac{1}{2}},
$$

the functions $A(p, s), B(p, s)$ are unknown and the branches in (20) are chosen such that the radicals have positive real parts.

We next consider the transformed heat fluxes along the line $y=0$, writing

$$
\bar{q}_{y j}(x, 0, s)= \begin{cases}0, & x<0, \\ m(x, s), & x>0,\end{cases}
$$

where $(21)_{1}$ follows from the boundary condition (12) and $m(x, s)$ is an unknown function to be determined We note that $q_{y j}(x, 0, t)$ must tend to the onedimensional result (3) at large $x$ and hence we anticipate that $m(x, s)$ tends to a constant as $x \rightarrow \infty$. Equations (21) have the following two-sided Laplace transform:

$$
\bar{q}_{y j}^{*}(p, 0, s)=M^{+}(p, s),
$$

where

$$
\left.\begin{array}{rl}
M^{+}(p, s) & =\int_{0}^{\infty} m(x, s) \exp \left(-s^{\frac{1}{2}} p x\right) d x, \\
m(x, s) & =\frac{s^{\frac{1}{2}}}{2 \pi i} \int_{p_{0}-i \infty}^{p_{0}+i \infty} M^{+}(p, s) \exp \left(s^{\frac{1}{t}} p x\right) d p .
\end{array}\right\}
$$

Clearly $\mathrm{M}^{+}(p, s)$ is a half-line transform, whereas $p_{0}$ is a real quantity, the bounds of which will be defined later.

In the same manner, we define the transforms $v_{j}(x, s), v_{j}^{-}(p, s)$ and $n(x, s)$, $N^{+}(p, s)$ of the temperatures along $y=0$ for $x<0$ and $x>0$, respectively. Further, we write $V^{-}(p, s) \equiv V_{1}^{-}(p, s)-V_{2}^{-}(p, s)$.

\section{The Wiener-Hopf problem}

Eliminating $A(p, s), B(p, s)$ and $N^{+}(p, s)$ from the transformed boundary conditions, we obtain the following functional equation of the Wiener-Hopf 
type:

$$
M^{+}(p, s)-L(p, s) T_{0} / s^{\mathbf{j}} p=-L(p, s) V^{-}(p, s),
$$

where the kernel is given by

$$
L(p, s)=\frac{K_{1} K_{2} \gamma_{1} \gamma_{2}}{K_{1} \gamma_{1}+K_{2} \gamma_{2}}
$$

We shall next perform a closed-form factorization of the above kernel. Some observations in the complex $p$-plane are in order. We first note that the function $m(x, s)$ has the behaviour

$$
m(x, s) \rightarrow \begin{cases}\text { constant, } & x \rightarrow+\infty, \\ 0, & x \rightarrow-\infty .\end{cases}
$$

This behaviour defines the strip of analyticity of the function $M^{+}(p, s)$ as well as the strip of convergence of the inverse Laplace transform in $(23)_{2}$. Since we anticipate a square-root singularity in heat flux as $x \rightarrow 0^{+}, y=0$ (see section 3 above), we also have

$$
M^{+}(p, s) \rightarrow p^{-\frac{1}{2}}, \quad|p| \rightarrow \infty .
$$

Secondly, for the functions $v_{j}(x, s)$, in view of equation (15), there must exist $c_{j}$ such that

$$
v_{j}(x, s)=O\left(\exp \left(s^{\frac{1}{2}} c_{j} x\right), \quad c_{j}>0, \text { as } x \rightarrow \infty,\right.
$$

which implies that the functions $V_{j}(p, s)$ are analytic in $\operatorname{Re}(p)<c_{j}$ and bounded as $|p| \rightarrow \infty$.

Finally, we should like to factorize the $\operatorname{kernel} L(p, s)$ into the product form

$$
L(p, s)=L^{+}(p, s) L^{-}(p, s),
$$

where $L^{+}(p, s)$ and $L^{-}(p, s)$ are functions that are analytic and non-zero to the right and to the left, respectively, of certain lines in the complex $p$-plane. Assuming that a suitable factorization of this form can be obtained, we can divide both sides of equation (24) by $L^{+}(p, s)$, with the result that

$$
\frac{M^{+}(p, s)}{L^{+}(p, s)}-L^{-}(p, s) \frac{T_{0}}{s^{\frac{1}{2}} p}=-L^{-}(p, s) V^{-}(p, s),
$$

which holds in a certain strip of the p-plane which will be defined later.

We also need to split the following term in the sum:

where by inspection

$$
L^{-}(p, s) \frac{T_{0}}{s^{i} p}=R^{+}(p, s)+R^{-}(p, s),
$$

$$
\left.\begin{array}{l}
R^{+}(p, s)=\frac{T_{0}}{s^{\frac{1}{2}} p} L^{-}(0, s), \\
R^{-}(p, s)=\frac{T_{0}}{s^{\frac{3}{2}} p}\left[L^{-}(p, s)-L^{-}(0, s)\right] .
\end{array}\right\}
$$


Clearly the crucial step is the factorization of the kernel. Equations (20), (25) can be combined to give

$$
L(p, s)=\frac{K_{2} s^{\frac{1}{t}}\left(b_{2}-p\right)^{\frac{1}{2}}\left(b_{2}+p\right)^{\frac{1}{2}}}{1+\lambda\left\{\left(b_{2}^{2}-p^{2}\right) /\left(b_{1}^{2}-p^{2}\right)\right\}^{\frac{1}{2}}},
$$

where we define the ratio of conductivities

$$
\lambda=K_{2} / K_{1} .
$$

The numerator in (33) can be split by inspection, whereas for the denominator we use Noble's treatise $(5$, p. 15 , Theorem C). In order to apply this theorem we observe that

$$
\left\{1+\lambda\left(\frac{b_{2}^{2}-p^{2}}{b_{1}^{2}-p^{2}}\right)^{\frac{1}{2}}\right\} \rightarrow(1+\lambda) \quad \text { as }|p| \rightarrow \infty .
$$

The kernel (33) can be written as

$$
L(p, s)=\frac{K_{2} s^{\frac{1}{2}}\left(b_{2}-p\right)^{\frac{1}{2}}\left(b_{2}+p\right)^{\frac{1}{2}}}{1+\lambda} \exp \{-\ln [v(p)]\},
$$

where

$$
v(p)=\frac{1+\lambda\left\{\left(b_{2}^{2}-p^{2}\right) /\left(b_{1}^{2}-p^{2}\right)\right\}^{\frac{1}{2}}}{1+\lambda}
$$

so that $v(p) \rightarrow 1$ as $|p| \rightarrow \infty$.

Then, referring to equation (29), we can write

$$
\left.\begin{array}{l}
L^{+}(p, s)=\frac{K_{2} s^{\frac{1}{2}}\left(b_{2}+p\right)^{\frac{1}{2}}}{1+\lambda} \exp \left\{+\frac{1}{2 \pi i} \int_{C_{t}} \ln [v(z)] \frac{d z}{z-p}\right\}, \\
L^{-}(p, s)=\left(b_{2}-p\right)^{\frac{1}{2}} \exp \left\{-\frac{1}{2 \pi i} \int_{C_{r}} \ln [v(z)] \frac{d z}{z-p}\right\},
\end{array}\right\}
$$

where the integration contours $C_{l}, C_{r}$ are shown in Fig. 2. The branch cuts introduced from $\left(-b_{2}, 0\right)$ to $\left(-b_{1}, 0\right)$ and from $\left(b_{1}, 0\right)$ to $\left(b_{2}, 0\right)$ are appropriate for the function $v(p)$. We have also assumed here that $b_{2}>b_{1}$ and hence that $\kappa_{1}>\kappa_{2}$. This involves no loss in generality, since the initial coordinate system can always be chosen to satisfy these inequalities.

Furthermore, since the integrand in (38) vanishes for $|z| \rightarrow \infty$, Cauchy's theorem and Jordan's lemma (6) imply that the original contours can be replaced by the contours $C_{l}^{\prime}, C_{r}^{\prime}$, wrapped around the left and the right branch cut respectively, as shown in Fig. 2. In order to determine the integrals, we must take into account that the function $\ln \{v(p)\}$ takes complex-conjugate values at the upper and lower surfaces of the branch cuts $\left(-b_{2},-b_{1}\right)$ and $\left(b_{1}, b_{2}\right)$. We also utilize the following property of logarithms:

$\ln \{v(p)\}=\ln \left\{\left[(\operatorname{Re} v(p))^{2}+(\operatorname{Im} v(p))^{2}\right]^{\ddagger}\right\}+i \tan ^{-1}\{\operatorname{Im} v(p) / \operatorname{Re} v(p)\}$. 


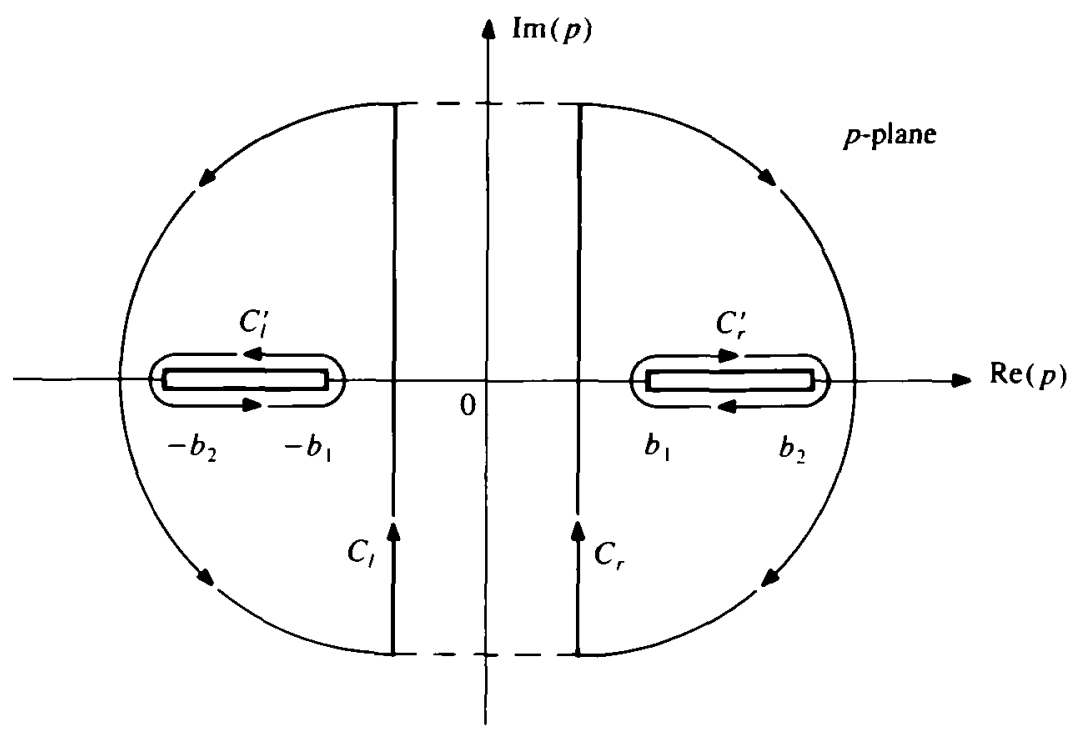

FIG. 2. Integration paths and branch cuts for the Wiener-Hopf kernel factorization

Thus, noting the form of the function $v(p)$ from equation (37), we write

$$
\tan ^{-1}\left\{\frac{\operatorname{Im} v(p)}{\operatorname{Re} v(p)}\right\}=\tan ^{-1}\left\{\lambda\left(\frac{b_{2}^{2}-p^{2}}{p^{2}-b_{1}^{2}}\right)^{\frac{1}{2}}\right\}
$$

and it is then straightforward to establish the following expressions for the split-kernel functions:

$$
\left.\begin{array}{l}
L^{+}(p, s)=\frac{K_{2} s^{\frac{1}{2}}\left(b_{2}+p\right)^{\frac{1}{2}}}{1+\lambda} \exp \left\{-\frac{1}{\pi} \int_{-b_{2}}^{-b_{1}} \tan ^{-1}\left(\lambda\left(\frac{b_{2}^{2}-z^{2}}{z^{2}-b_{1}^{2}}\right)^{\frac{1}{2}}\right) \frac{d z}{z-p}\right\}, \\
L^{-}(p, s)=\left(b_{2}-p\right)^{\frac{1}{2}} \exp \left\{\frac{1}{\pi} \int_{b_{1}}^{b_{2}} \tan ^{-1}\left(\lambda\left(\frac{b_{2}^{2}-z^{2}}{z^{2}-b_{1}^{2}}\right)^{\frac{1}{2}}\right) \frac{d z}{z-p}\right\},
\end{array}\right\}
$$

where the functions $L^{+}(p, s)$ and $L^{-}(p, s)$ are analytic and non-zero in the half-planes $\operatorname{Re}(p)>-b_{1}$ and $\operatorname{Re}(p)<b_{1}$ respectively.

Having now obtained the required factorization in equation (29), and taking into account (31), (32), we can write the Wiener-Hopf equation in the following form:

$$
\begin{aligned}
\frac{M^{+}(p, s)}{L^{+}(p, s)}-\frac{T_{0}}{s^{p} p} L^{-}(0, s) & =-L^{-}(p, s) V^{-}(p, s)+\frac{T_{0}}{s^{d} p}\left[L^{-}(p, s)-L^{-}(0, s)\right] \\
& \equiv J(p, s) .
\end{aligned}
$$


This equation holds in the strip $0<\operatorname{Re}(p)<\inf \left(c_{j}, b_{1}\right)$. It can then be proved that $J(p, s)=0$. To this end, we follow the methodology described by Noble (5, pp. 36-38), utilizing the known asymptotic behaviour of the functions in equation (42).

Working on the first member (on the left-hand side) of equation (42), we recall (27) and further note from $(41)_{1}$ that $L^{+}(p, s) \rightarrow p^{\frac{1}{2}}$ for $|p| \rightarrow \infty$. Thus, the first member of (42) tends to zero as $|p| \rightarrow \infty$. This is also true for the second member of (42).

So far, equation (42) defines $J(p, s)$ only in the strip $0<\operatorname{Re}(p)<\inf \left(c_{j}, b_{1}\right)$, but the first part is analytic in $\operatorname{Re}(p)>0$ and the second in $\operatorname{Re}(p)<\inf \left(c_{j}, b_{1}\right)$. Therefore, by analytic continuation, we can define $J(p, s)$ over the whole $p$-plane. Then, by taking into account the above-mentioned asymptotic behaviour at infinity and by the extended Liouville theorem, we conclude that $J(p, s)=0$.

It then follows from (42) that

$$
M^{+}(p, s)=\frac{T_{0}}{s^{\frac{3}{2}} p} L^{-}(0, s) L^{+}(p, s) .
$$

The temperature field inside the two bodies $(y \neq 0)$ can now be obtained using equation (19) and performing a double Laplace-transform inversion, following the method of the next section. However, we restrict attention here to the heat flux at the interface, $q_{y j}(x, 0, t)$, which is the quantity of the most physical interest. We also recall in passing that the temperature is bounded at the origin, being given by equation (19), but that the heat flux has a square-root singularity (see section 2 above).

\section{Laplace-transform inversion}

From equations (21) to (23), we note that

$$
\bar{q}_{y j}(x, 0, s)=\frac{s^{\frac{1}{2}}}{2 \pi i} \int_{p_{0}-i \infty}^{p_{0}+\infty} M^{+}(p, s) \exp \left(s^{\frac{1}{t}} p x\right) d p,
$$

where the integration path is shown in Fig. 3. We shall try the double Laplace transform at the same time by following the Cagniard-de Hoop method, which was developed for elastodynamic problems (7). The central idea in the next step is to convert the integral in (44) to a form which permits inversion of the one-sided Laplace transform on time by inspection.

Equations (43), (44) give

$$
\bar{q}_{y j}(x, 0, s)=\frac{T_{0} L^{-}(0, s)}{2 \pi i s} \int_{p_{0}-i \infty}^{p_{0}+i_{\infty}} \frac{L(p, s) \exp \left(s^{\frac{1}{t}} p x\right)}{p L^{-}(p, s)} d p,
$$

where we have replaced $L^{+}(p, s)$ by $L(p, s) / L^{-}(p, s)$, since the former function is not analytic in the left half-plane, where we intend to deform the integration path. Then, the only singularities of the integrand in equation (45) in the half-plane $\operatorname{Re}(p)<\inf \left(c_{j}, b_{1}\right)$ are a pole at $p=0$ and the branch points of 


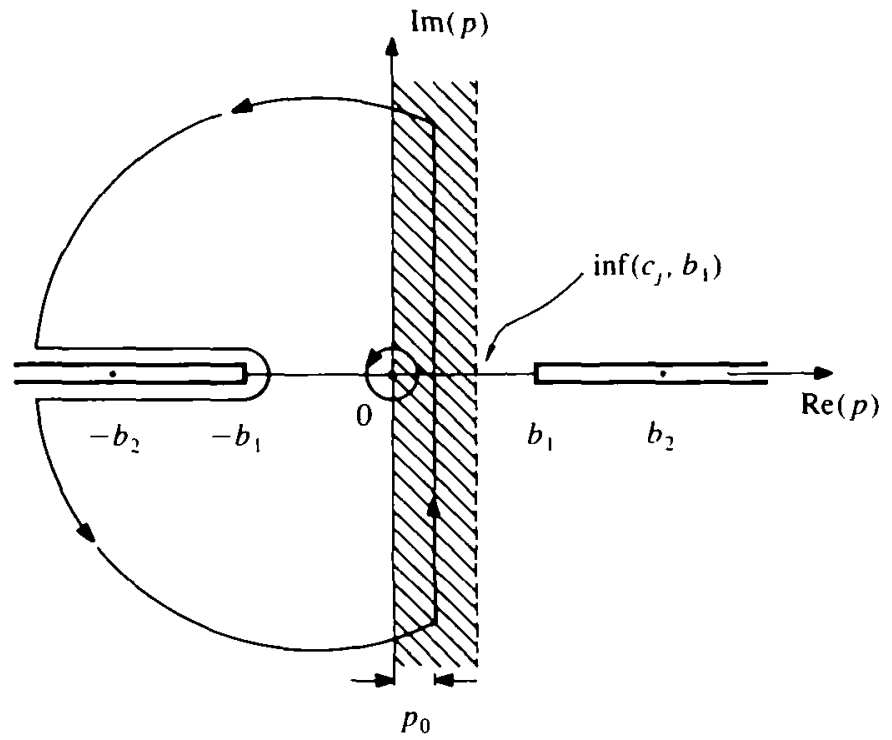

FIG. 3. Strip of convergence and auxiliary paths for Laplace-transform inversion

$L(p, s)$ at $p=-b_{1},-b_{2}$ (recall that $L^{-}(p, s)$ is analytic in that half-plane).

We now deform the integration path so that the integral in (45) takes the form of a Laplace transform. By invoking Cauchy's theorem, we have that the integral along a path consisting of the vertical line from $\left(p_{0}-i \infty\right)$ to $\left(p_{0}+i \infty\right)$, the branch-cut line and the two remote arcs of infinite radius, is equal to the residue of the integrand at the pole. The contribution from the remote arcs is clearly zero and hence equation (45) becomes

$$
\bar{q}_{y j}=-\frac{T_{0} L^{-}(0, s)}{2 \pi i s}\left\{\left(I_{u p}+I_{l o}\right)-2 \pi i(\operatorname{Res})\right\},
$$

where

$$
I_{u p}=\int_{-\infty}^{-b_{1}} \frac{L(p, s) \exp \left(s^{\frac{1}{2}} p x\right)}{p L^{-}(p, s)} d p, \quad I_{l o}=\int_{-b_{1}}^{-\infty} \frac{L(p, s) \exp \left(s^{\frac{1}{2}} p x\right)}{p L^{-}(p, s)} d p
$$

and $\operatorname{Res}$ is the residue of the integrand at $p=0$, given by

$$
\operatorname{Res}\left\{\frac{L(p, s) \exp \left(s^{\frac{t}{2}} p x\right)}{p L^{-}(p, s)}\right\}=\frac{K_{2} b_{1} b_{2} s^{\text {t }}}{\left(b_{1}+\lambda b_{2}\right) L^{-}(0, s)} .
$$

An immediate observation at this point is that as $p$ approaches the negative real axis, we have

$$
p \rightarrow-t^{\frac{1}{2}} / x, \quad x, t>0,
$$

a fact which we shall utilize later. 
In order to calculate the branch-line integral in (47), we shall exploit the fact that the function $L(p, s)$ takes complex-conjugate values at the top and bottom of the cut $\left(-\infty,-b_{1}\right)$. In view of this, equation (46) can be written in the form

$$
\begin{aligned}
\bar{q}_{y j}(x, 0, s)= & \frac{T_{0} L^{-}(0, s)}{\pi s^{\frac{1}{t}}}\left\{\int_{-\infty}^{-b_{2}} \frac{K_{2}\left(p^{2}-b_{2}^{2}\right)^{\frac{1}{2}}}{\left[1+\lambda\left\{\left(p^{2}-b_{2}^{2}\right) /\left(p^{2}-b_{1}^{2}\right)\right\}^{\frac{1}{1}}\right]} \frac{\exp \left(s^{\frac{1}{2}} p x\right)}{p L^{-}(p, s)} d p\right. \\
& \left.+\int_{-b_{2}}^{-b_{1}} \frac{K_{2} \lambda\left(b_{2}^{2}-p^{2}\right)\left(p^{2}-b_{1}^{2}\right)^{-\frac{1}{2}}}{\left[1+\lambda^{2}\left\{\left(b_{2}^{2}-p^{2}\right) /\left(p^{2}-b_{1}^{2}\right)\right\}\right]} \frac{\exp \left(s^{\frac{1}{2}} p x\right)}{p L^{-}(p, s)} d p\right\} \\
& +\frac{T_{0} K_{2} b_{1} b_{2}}{\left(b_{1}+\lambda b_{2}\right) s^{\frac{1}{t}}} \\
\equiv & \bar{q}_{y j}^{\text {pert }}+\bar{q}_{y j}^{\text {const }} .
\end{aligned}
$$

The second term in these equations is independent of $p$ and hence corresponds to a term in the untransformed heat flux which is independent of the space coordinate $x$. In fact, we shall show below that it represents the onedimensional heat flux of equation (3) above. The first term clearly depends upon both space and time and represents the perturbation in the onedimensional heat flux due to the mixed boundary conditions of the problem.

The term $s^{-\frac{1}{2}}$ appearing in equation (50) can be written in two alternative forms in order to facilitate the inversion of $q_{y j}(x, 0, s)$. We have

$$
\begin{aligned}
s^{-\frac{1}{2}} & =\int_{0}^{\infty} \exp \left(-s^{\frac{1}{t}} t\right) d t \\
& =\int_{0}^{\infty}(\pi t)^{-\frac{1}{2}} \exp (-s t) d t .
\end{aligned}
$$

Substitution of $(52 b)$ into the second term of equation (50) enables the inversion to be performed by inspection, with the result that

$$
q_{y j}^{\text {const }}=\frac{T_{0} K_{2} b_{1} b_{2}}{\left(b_{1}+\lambda b_{2}\right)}(\pi t)^{-\frac{1}{t}}
$$

which agrees with the one-dimensional result (3) as expected.

For the first term in (50), we use the form (52a) and enforce (49), obtaining

where

$$
\bar{q}_{y j}^{\text {pert }}(x, 0, s)=\frac{T_{0} b_{2}^{t}}{\pi} \int_{0}^{\infty} \exp \left(-s^{\frac{1}{t}} t\right) d t \int_{0}^{\infty} F(x, u) \exp \left(-s^{\frac{1}{2}} u\right) d u,
$$

$$
F(x, u)= \begin{cases}0, & 0<u<b_{1} x, \\ \frac{K_{2} \lambda\left(u^{2}-b_{1}^{2} x^{2}\right)^{\frac{1}{1}}\left(b_{2}^{2} x^{2}-u^{2}\right)}{\left[\left(u^{2}-b_{1}^{2} x^{2}\right)+\lambda^{2}\left(b_{2}^{2} x^{2}-u^{2}\right)\right]} \frac{\exp (\Psi)}{x^{\frac{1}{2}} u\left(b_{2} x+u\right)^{\frac{1}{2}}}, & b_{1} x<u<b_{2} x, \\ \frac{K_{2}\left(u^{2}-b_{1}^{2} x^{2}\right)^{\frac{1}{1}}\left(u^{2}-b_{2}^{2} x^{2}\right)}{\left[\left(u^{2}-b_{1}^{2} x^{2}\right)^{\frac{1}{2}}+\lambda\left(u^{2}-b_{2}^{2} x^{2}\right)^{\frac{1}{t}}\right]} \frac{\exp (\Psi)}{x^{\frac{1}{2}} u\left(b_{2} x+u\right)^{\frac{1}{4}}}, & b_{2} x<u<\infty,\end{cases}
$$


and

$$
\Psi=\frac{u}{\pi x} \int_{b_{1}}^{b_{2}} \tan ^{-1}\left\{\lambda\left(\frac{b_{2}^{2}-z^{2}}{z^{2}-b_{1}^{2}}\right)^{\frac{1}{2}}\right\} \frac{d z}{z(z+u / x)} .
$$

We can interchange the order of integration in (54) obtaining

$$
\bar{q}_{y j}^{\text {pert }}(x, 0, s)=\frac{T_{0} b_{2}^{t}}{\pi} \int_{0}^{\infty}\left\{\int_{0}^{\xi} F(x, u) d u\right\} \exp \left(-s^{\frac{1}{2}} \xi\right) d \xi .
$$

We now establish an operational rule for the case where $s$ is replaced by $s^{\frac{1}{2}}$ in the usual Laplace transform, since this is required to invert equation (57). From tables of Laplace transforms (for example, $(8)$ ), we have the result

$$
\exp \left(-u s^{\frac{1}{2}}\right)=\int_{0}^{\infty}\left\{\frac{u}{2\left(\pi t^{3}\right)^{\frac{3}{2}}} e^{-w^{2} / 4 t}\right\} e^{-s t} d t,
$$

where $u>0$. Multiplying both sides of $(58)$ by the function $h(u)$ and integrating from zero to infinity, we obtain

$$
\int_{0}^{\infty} h(u) \exp \left(-u s^{\frac{1}{2}}\right) d u=\int_{0}^{\infty}\left\{\frac{1}{2\left(\pi t^{3}\right)^{\frac{3}{2}}} \int_{0}^{\infty} u h(u) e^{-u^{2} / 4 t} d u\right\} e^{-s t} d t
$$

which is a general result. We now write

$$
h(x, \xi)=\int_{0}^{\xi} F(x, u) d u
$$

which in combination with equation (59) permits us to rewrite (57) in the form

$$
\bar{q}_{y j}^{\text {pert }}(x, 0, s)=\frac{T_{0} b_{2}^{\frac{1}{2}}}{\pi} \int_{0}^{\infty}\left\{\frac{1}{2\left(\pi t^{3}\right)^{\frac{3}{2}}} \int_{0}^{\infty} u h(x, u) e^{-x^{2} / 4 t} d u\right\} e^{-\boldsymbol{x} t} d t
$$

The one-sided Laplace transform can now be inverted by inspection with the result

$$
\begin{aligned}
q_{y j}^{\text {pert }}(x, 0, t) & =\frac{T_{0} b^{\frac{1}{2}}}{2(\pi t)^{\frac{1}{1}}} \int_{0}^{\infty} u h(x, u) e^{-u^{2} / 4 t} d u \\
& =\frac{T_{0} b^{\frac{1}{2}}}{2(\pi t)^{\frac{1}{2}}} \int_{0}^{\infty} u e^{-u^{2} / 4 t} \int_{0}^{\nu} F(x, w) d w d u
\end{aligned}
$$

Finally, interchanging the order of integration in (63), performing the inner integral and using the result $F(x, u)=0$ for $0<u<b_{1} x$, we get

$$
q_{y j}^{\text {pert }}=\frac{T_{0} b^{\frac{3}{2}}}{\left(\pi^{3} t\right)^{\frac{1}{3}}} \int_{b_{1} x}^{\infty} F(x, w) e^{-w^{2} / 4 t} d w .
$$




\section{Heat exchange between the bodies in contact}

We can now assemble the complete expression for the heat flux between the bodies at the interface $y=0, x>0$, using equations (51), (53), (64), with the result that

$$
q_{y j}(x, 0, t)=\frac{T_{0} K_{2} b_{1} b_{2}}{\left(b_{1}+\lambda b_{2}\right)}(\pi t)^{-\frac{1}{2}}+\frac{T_{0} b_{2}^{\frac{t}{2}}}{\left(\pi^{3} t\right)^{\frac{1}{1}}} \int_{b_{1} x}^{\infty} F(x, w) e^{-w^{2} / 4 t} d w,
$$

where the function $F(x, w)$ is defined by equations (55).

It is convenient at this stage to present the results in a dimensionless formulation, thus exposing the dependence of the solution on the parameter

$$
X=x /\left(4 \kappa_{1} t\right)^{\frac{1}{2}}=b_{1} x /\left(2 t^{\frac{1}{2}}\right) .
$$

We define the dimensionless integration variables

$$
\tau=w /\left(b_{1} x\right), \quad \rho=z / b_{1}
$$

and the ratios of material properties

$$
\mu=b_{2} / b_{1}=\left(\kappa_{1} / \kappa_{2}\right)^{\frac{1}{2}}, \quad \lambda=K_{2} / K_{1} .
$$

Substituting these expressions into equation (65), we obtain

$$
\begin{aligned}
q_{y j}(x, 0, t)= & \frac{T_{0} K_{2} b_{1} b_{2}}{\left(b_{1}+\lambda b_{2}\right)(\pi t)^{\frac{1}{2}}} \\
& +\frac{T_{0} K_{2}\left(b_{1} b_{2}\right)^{\frac{1}{2}}}{\left(\pi^{3} t\right)^{\frac{1}{2}}}\left\{\int_{1}^{\mu} \frac{\lambda\left(\tau^{2}-1\right)^{\frac{1}{1}}\left(\mu^{2}-\tau^{2}\right) \exp (\Psi) e^{-\tau^{2} X^{2}}}{\left[\left(\tau^{2}-1\right)+\lambda^{2}\left(\mu^{2}-\tau^{2}\right)\right] \tau(\mu+\tau)^{\frac{1}{2}}} d \tau\right. \\
& \left.+\int_{\mu}^{\infty} \frac{\left(\tau^{2}-1\right)^{\frac{1}{2}}\left(\tau^{2}-\mu^{2}\right)^{\frac{1}{2}} \exp (\Psi) e^{-\tau^{2} X^{2}}}{\left[\left(\tau^{2}-1\right)^{\frac{1}{2}}+\lambda\left(\tau^{2}-\mu^{2}\right)^{\frac{1}{2}}\right] \tau(\mu+\tau)^{\frac{1}{4}}} d \tau\right\},
\end{aligned}
$$

where

$$
\Psi=\frac{\tau}{\pi} \int_{1}^{\mu} \tan ^{-1}\left\{\lambda\left(\frac{\mu^{2}-\rho^{2}}{\rho^{2}-1}\right)^{\frac{1}{2}}\right\} \frac{d \rho}{\rho(\rho+\tau)} .
$$

Notice that the integral in equation (69) depends only on $X$ and the material properties. The dependence on $t$ is restricted to an explicit multiplier of $t^{-\frac{1}{t}}$ on each term.

\section{The total heat flux}

As explained in section 2 above, the short-time heat flux between two bodies which make contact over an arbitrary region of the plane $y=0$ only differs from the one-dimensional result (3) at points which are close to the boundary of the contact region. The thickness of this boundary layer grows with $t^{\frac{1}{2}}$, and hence will be small in comparison with the characteristic lengths of the contact region (in particular the minimum radius of curvature of the boundary) at small 
values of $t$. The temperature field will then be locally two-dimensional and the additional heat flux adjacent to the boundary will therefore be given by the term $q_{y j}^{\text {pert }}$ in the preceding analysis - that is, by the second term in equation (69), where $x$ is now to be interpreted as the distance from a given point in the contact region to the nearest point on the boundary.

We can now integrate the heat flux over the contact area to obtain an expression for the total heat flux as a function of time. The first term in equation (69), corresponding to the one-dimensional solution of equation (3), is uniform throughout the contact region and hence contributes a term proportional to the total contact area $A$. The second term is significant only in the boundary layer, which is thin in comparison with the radius of curvature of the boundary. Each element $d S$ of the perimeter $S$ of the contact region therefore makes a contribution to the total heat flux proportional to the integral of the perturbation term. $\dagger$

The total heat flux is thus given by

$$
Q=\frac{T_{0} K_{2} b_{1} b_{2} A}{\left(b_{1}+\lambda b_{2}\right)(\pi t)^{\frac{t}{2}}}+Q_{s} S,
$$

where

$$
Q_{s}=\int_{0}^{\infty} q_{y j}^{\text {pert }}(x, 0, t) d x
$$

Substituting for $q_{y j}^{\text {pert }}$ from the second term in equation (69), interchanging the order of integration and performing the inner integral, we obtain

$$
Q_{s}=T_{0} K_{2} f(\lambda, \mu)
$$

where

$$
\begin{aligned}
f(\lambda, \mu)= & \frac{\mu^{\frac{1}{2}}}{\pi}\left\{\int_{1}^{\mu} \frac{\lambda\left(\tau^{2}-1\right)^{\frac{1}{2}}\left(\mu^{2}-\tau^{2}\right) \exp (\Psi)}{\left[\left(\tau^{2}-1\right)+\lambda^{2}\left(\mu^{2}-\tau^{2}\right)\right] \tau^{2}(\mu+\tau)^{\frac{1}{2}}} d \tau\right. \\
& \left.+\int_{\mu}^{\infty} \frac{\left(\tau^{2}-1\right)^{\frac{1}{2}}\left(\tau^{2}-\mu^{2}\right)^{\frac{1}{2}} \exp (\Psi)}{\left[\left(\tau^{2}-1\right)^{\frac{1}{2}}+\lambda\left(\tau^{2}-\mu^{2}\right)^{\frac{1}{2}}\right] \tau^{2}(\mu+\tau)^{\frac{1}{2}}} d \tau\right\},
\end{aligned}
$$

and hence equation (71) can be written as

$$
Q=\frac{T_{0} K_{2} b_{1} b_{2} A}{\left(b_{1}+\lambda b_{2}\right)(\pi t)^{\frac{t}{t}}}+T_{0} K_{2} f(\lambda, \mu) S .
$$

In the special case where the materials have equal diffusivities, $\mu=1$ and

\footnotetext{
† This statement requires some qualification if the boundary of the contact region has corner points, at which the temperature field will be locally three-dimensional This question is discussed in more detail in section 9 below.
} 
equation (74) reduces to

$$
f(\lambda, 1)=\frac{1}{\pi(1+\lambda)} \int_{1}^{\infty} \frac{(\tau-1)^{\frac{1}{2}}}{\tau^{2}} d \tau=\frac{1}{2(1+\lambda)}
$$

see for example $(9,2.225 .2)$. We therefore get

$$
Q=\frac{T_{0} K_{2} b_{1} b_{2} A}{\left(b_{1}+\lambda b_{2}\right)(\pi t)^{\frac{1}{t}}}+\frac{T_{0} K_{2} S}{2(1+\lambda)},
$$

which agrees with the result of (2) when $\lambda=1$ and hence $K_{2}=K_{1}$. This agreement provides a useful check on the preceding analysis and also incidentally constitutes an analytical proof that the constant $C$ in (2, equation (31)) is indeed $\pi / 4$, a result which was only surmised on the basis of numerical integration in that paper. (Note that in (2, equation (31)), $T_{0}$ denotes the temperature of the interfacial plane, $y=0$, which will be $T_{0} / 2$ for similar materials with the present notation.)

\section{The effect of corner points}

If the contact region $A$ has corner points, the temperature field will be locally three-dimensional and hence the local heat flux will not be accurately represented by equation (65) at points whose distance from the corner is within the range defined by (5). Furthermore, even if (65) were taken to be a good approximation to the local heat flux, (71) would be incorrect for its integral, since as shown in Fig. 4, we need to exclude a triangular region of the boundary layer associated with each edge from the domain of integration for the perturbation term $q_{y j}^{\text {pert }}$.

The error due to the domain of integration can be written as

$$
\Delta Q_{1}=2 \int_{0}^{\infty} q_{y j}^{\text {pert }} x \tan \alpha d x
$$

where $2 \alpha$ is the external angle at the corner.

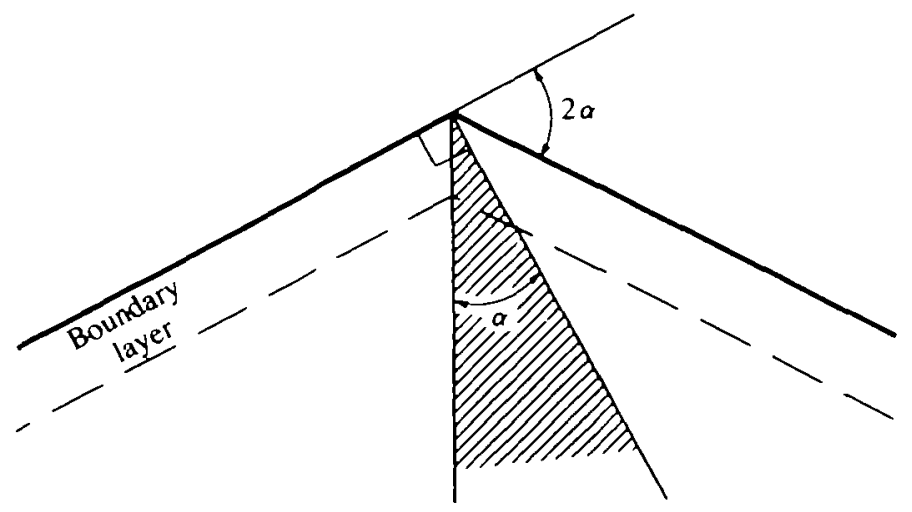

FIG. 4. Geometry of the boundary layer at a corner. The shaded area is the domain of integration in equation (84). 
Substituting for $q_{y j}^{\text {pert }}$ from (64), this result can be written in the form

$$
\Delta Q_{1}=\frac{T_{0} K_{2} t^{\frac{1}{2}}}{b_{1}} g(\lambda, \mu, \alpha),
$$

where $g(\lambda, \mu, \alpha)$ is a dimensionless function.

We can probe the three-dimensional nature of the local temperature field by considering the heat-conduction problem of two half-spaces, with the same initial conditions as in section 3 , where the contact region is the semi-infinite sector $0<\theta<\alpha, r>0, y=0$ in polar coordinates centred on the corner in the plane of the interface.

For this problem, we anticipate that a two-dimensional boundary layer will be found adjacent to the boundary lines $\theta=0, \alpha$, but that three-dimensional effects will be significant roughly in the region $0<r<s$, where $s$ is the thickness of the two-dimensional boundary layer.

The problem is self-similar and hence the temperature field must depend only on the dimensionless coordinates $X=b_{1} x / \sqrt{ }(4 t)$, etc. - that is, $T=T(X, Y, Z)$. The local heat flux at the surface can then be written as

$$
q_{y 1}\left(x, 0^{-}, z, t\right)\left\{\begin{array}{l}
=-K_{2} \frac{\partial T}{\partial y}, \quad y=0^{-}, \\
=-\frac{b_{1} K_{2}}{\sqrt{ }(4 t)} \frac{\partial T(X, Y, Z)}{\partial Y}, \quad Y=0^{+}, \\
=-\frac{b_{1} K_{2}}{\sqrt{ }(4 t)} F(X, Z),
\end{array}\right.
$$

where $F$ is a function of the dimensionless coordinates $X, Z$ only.

We next subtract out the heat flux predicted from the two-dimensional analysis (equation (69)), leaving a quantity $q_{y 1}^{*}=q_{y_{1}}-q_{y 1}^{\text {pert }}$ which decays to zero as $r \rightarrow \infty$. Dimensional arguments show that $q^{*}$ can also be written in the form

$$
q_{y}^{*}(x, 0, z, t)=-\frac{b_{1} K_{1}}{\sqrt{ }(4 t)} F^{*}(X, Z) .
$$

Finally, we integrate this heat flux over the sector $0<\theta<\alpha, r>0$ to determine the correction to the total heat exchange associated with the corner point, with the result that

$$
\begin{aligned}
\Delta Q_{2} & =\int_{A} q_{y}^{*}(x, 0, z, t) d x d z \\
& =-b_{1} K_{2} \sqrt{ }(4 t) \int_{A} F^{*}(X, Z) d X d Z,
\end{aligned}
$$


where $A$ now denotes the sector $0<\theta<\alpha, r>0$. This expression is of the form

$$
\Delta Q_{2}=-b_{1} K_{2} t^{\frac{t}{t}} h(\lambda, \mu, \alpha),
$$

where $h(\lambda, \mu, \alpha)$ is a dimensionless function depending on the material properties and the angle of the corner only.

The correction for the corner is $\Delta Q=\Delta Q_{2}-\Delta Q_{1}$ and we can therefore deduce from dimensional considerations alone that the correction will consist of a single term proportional to $t^{\frac{1}{2}}$, which is of higher order in $t$ than the two terms of equation (71). Beck et al. (10) give corrections for both corner points and curvature of the edge of the heated region in their solution of the related unmixed boundary-value problem for the mean temperature of a region subjected to uniform heating.

\section{Numerical results}

To apply equation (75) to contact problems involving more general material combinations, we need to evaluate the function $f(\lambda, \mu)$ from equation (74). The

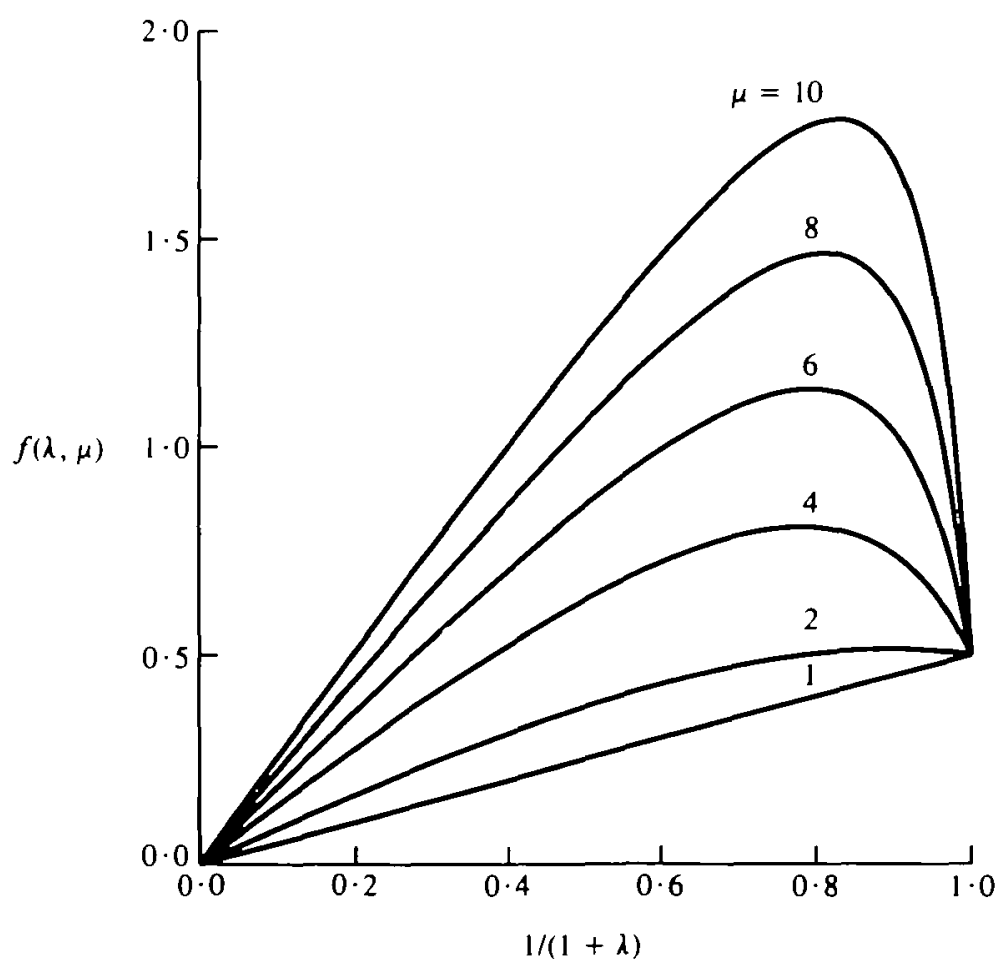

FIG. 5. The function $f(\lambda, \mu)$ determining the coefficient of the second term in the asymptotic expression for the short-time heat flux between dissimilar bodies (see equation (75)) 


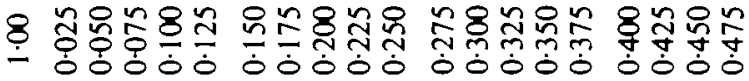

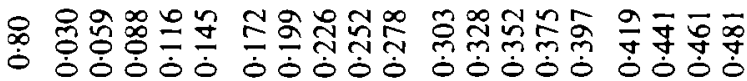

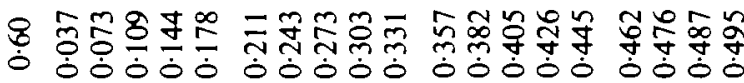

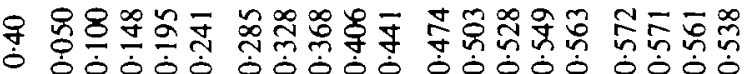

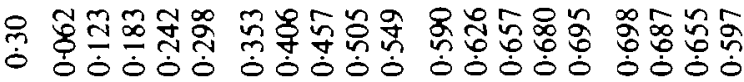

₹ $\equiv$

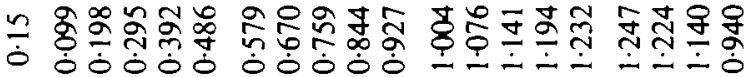

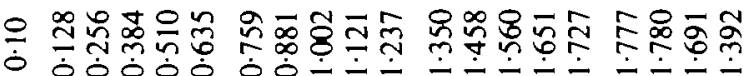

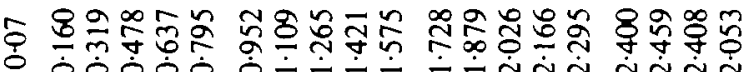

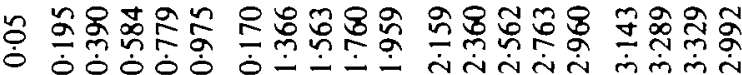

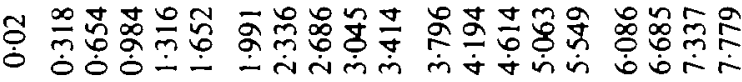

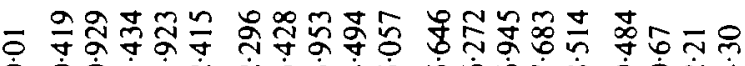

$\dot{\partial} \dot{\underline{U}} \dot{\dot{X}}$

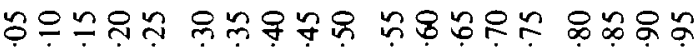

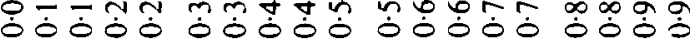

$$
- \pm
$$


authors have been unable to find a closed-form expression for this integral in the general case, but it presents no particular challenge to numerical evaluation. Also, since the function depends on only two parameters, fairly comprehensive results can be obtained.

We recollect that the materials are labelled so as to ensure that $\mu>1$, whereas there is no restriction on the conductivity ratio $\lambda$, except that it be positive. For the presentation of the numerical results, it is convenient to condense the infinite range on $\lambda$, by plotting $f(\lambda, \mu)$ as a function of $1 /(1+\lambda)$ for various values of $\mu$, as shown in Fig. 5. Corresponding numerical results are given in Table 1 .

\section{Conclusions}

The above solution describes the nature of the temperature and heat-flux fields in the immediate vicinity of the boundary of a recently established contact region between two conducting bodies at different initial temperatures. It also permits the development of a short-time asymptotic expression for the heat flux between two such bodies. This expression (equation (75)) depends only upon the area and perimeter of the contact region and hence can be applied to a region of any shape.

\section{Acknowledgements}

The authors are pleased to acknowledge support from the National Science Foundation under contract MSM-8711557. The second author also acknowledges a useful discussion of this problem with Professor G. M. L. Gladwell.

\section{REFERENCES}

1. H. Carslaw and J. C. Jaeger, The Conduction of Heat in Solids, 2nd edition (Clarendon Press, Oxford 1959).

2. J. R. BARBer, Int. J. Heat Mass Transfer 32 (1989) 943-949.

3. M. L. Williams, J. appl. Mech. 19 (1952) 526-528.

4. B. VAN DER POL and H. Bremmer, Operational Calculus Based on the Two-Sided Laplace Integral (University Press, Cambridge 1950).

5. B. NoBle, Methods Based on the Wiener-Hopf Technique (Pergamon Press, New York 1958).

6. E. T. Whittaker and G. N. Watson, A Course of Modern Analysis (University Press, Cambridge 1980).

7. A. T. DE HoOp, Appl. Sci. Res. 8 (1960) 349-356.

8. R. V. ChurchiLl, Operational Mathematics (McGraw-Hill Kogakusha, Tokyo 1972).

9. I. S. Gradshteyn and I. M. Ryzhik, Table of Integrals, Series and Products (Academic Press, New York 1980).

10. J. V. Beck, N. R. Keltner and I. P. Schisler, Al AA J. 23 (1985) 1978-1982. 